\title{
Optimal Multimodal Fusion for Multimedia Data Analysis
}

\author{
Yi Wu \\ Dept. Computer Engineering \\ University of California Santa Barbara \\ Santa Barbara, CA 93106 \\ wuyi@ece.ucsb.edu \\ Kevin Chen-Chuan Chang
Dept. Computer Science \\ University of Illinois at Urbana-Champaign \\ Urbana, IL 61801 \\ kcchang@cs.uiuc.edu
}

\author{
Edward Y. Chang \\ Dept. Computer Engineering \\ University of California Santa Barbara \\ Santa Barbara, CA 93106 \\ echang@ece.ucsb.edu
}

\author{
John R. Smith \\ IBM T.J. Watson Research Center \\ 19 Skyline Drive \\ Hawthorne, NY 10532 \\ jrsmith@watson.ibm.com
}

\begin{abstract}
Considerable research has been devoted to utilizing multimodal features for better understanding multimedia data. However, two core research issues have not yet been adequately addressed. First, given a set of features extracted from multiple media sources (e.g., extracted from the visual, audio, and caption track of videos), how do we determine the best modalities? Second, once a set of modalities has been identified, how do we best fuse them to map to semantics? In this paper, we propose a two-step approach. The first step finds statistically independent modalities from raw features. In the second step, we use super-kernel fusion to determine the optimal combination of individual modalities. We carefully analyze the tradeoffs between three design factors that affect fusion performance: modality independence, curse of dimensionality, and fusion-model complexity. Through analytical and empirical studies, we demonstrate that our two-step approach, which achieves a careful balance of the three design factors, can improve class-prediction accuracy over traditional techniques.
\end{abstract}

Categories and Subject Descriptors: H.3.1 [INFORMATION STORAGE AND RETRIEVAL]: Content Analysis and Indexing

General Terms: Algorithms

Keywords: Multimodal fusion, independent analysis, super-kernel fusion, modality independence, curse of dimensionality

\section{INTRODUCTION}

Multimedia data such as images and videos are represented by features from multiple media sources. Traditionally, images are represented by keywords and perceptual features such as color, texture, and shape [25]. Videos are represented by features embedded

Permission to make digital or hard copies of all or part of this work for personal or classroom use is granted without fee provided that copies are not made or distributed for profit or commercial advantage and that copies bear this notice and the full citation on the first page. To copy otherwise, to republish, to post on servers or to redistribute to lists, requires prior specific permission and/or a fee.

MM'04, October 10-16, 2004, New York, New York, USA

Copyright 2004 ACM 1-58113-893-8/04/0010 ...\$5.00. in the visual, audio and caption tracks. [2]. These features are extracted and then fused in a complementary way for understanding the semantics of multimedia data [21].

Unfortunately, traditional work on multimodal integration has largely been heuristic-based. It lacks theories to answer two fundamental questions: 1) what are the best modalities? and 2) how can we optimally fuse information from multiple modalities? Suppose we extract $l, m, n$ features from the visual, audio, and caption tracks of videos. At one extreme, we could treat all these features as one modality and form a feature vector of $l+m+n$ dimensions. At the other extreme, we could treat each of the $l+m+n$ features as one modality. We could also regard the extracted features from each media-source as one modality, formulating a visual, audio, and caption modality with $l, m$, and $n$ features, respectively. Almost all prior multimodal-fusion work in the multimedia community employs one of these three approaches. But, can any of these feature compositions yield the optimal result?

Statistical methods such as principle component analysis (PCA) and independent component analysis (ICA) have been shown to be useful for feature transformation and selection. PCA is useful for denoising data, and ICA aims to transform data to a space of independent axes (components). Despite their best attempt under some error-minimization criteria, PCA and ICA do not guarantee to produce independent components. In addition, the created feature space may be of very high dimensions and thus be susceptible to the curse of dimensionality ${ }^{1}$. In the first part of this paper, we propose an independent modality analysis scheme, which identifies independent modalities, and at the same time, avoids the curse-ofdimensionality challenge.

Once a good set of modalities has been identified, the second research challenge is to fuse these modalities in an optimal way to perform data analysis (e.g., classification). Suppose we can yield truly independent modalities, and each modality can derive accurate posterior probability for class prediction. We can simply use the product-combination rule to multiply the probabilities for pre-

\footnotetext{
${ }^{1}$ The work of [7] shows that, when data dimension is high, the distances between pairs of objects in the space become increasingly similar to each other due to the central limit theory. This phenomenon is called the dimensionality curse [6], because it can severely hamper the effectiveness of data analysis.
} 
dicting class membership. Unfortunately, the above two conditions do not hold in general for a multimedia data-analysis task (see Section 2 for detailed discussion). Using the product-combination rule to fuse information is thus inappropriate. Another popular fusion method is the weighted-sum rule, which performs a linear combination on the modalities. The weighted-sum rule enjoys the advantage of simplicity, but its linear constraint forbids high model complexity; hence it cannot adequately explore the interdependencies left unresolved by PCA and ICA. We propose our super-kernel fusion scheme to fuse individual modalities in a non-linear way (linear fusion is a special case of our method). The super-kernel fusion scheme finds the best combination of modalities through supervised training.

\subsection{An Illustrative Example}

Let us use a simple example to explain the shortcomings of some traditional multimodal integration schemes that invite further research. Figure 1 shows the existence of feature dependencies in a real image dataset, before and after performing PCA/ICA. This figure plots the normalized correlation matrix in absolute value derived from a $2 k$-image dataset of 14 classes. (Detailed description for this image dataset is given in Section 5.) A total of 144 features are considered: the first 108 are color features; the other 36 are texture features. Correlation between features within the same media source and across different media sources is measured by computing the covariance matrix:

$$
C=\frac{1}{N} \sum_{\mathbf{x}_{i} \in X}\left(\mathbf{x}_{i}-\overline{\mathbf{x}}\right)\left(\mathbf{x}_{i}-\overline{\mathbf{x}}\right)^{T} \text { with } \overline{\mathbf{x}}=\frac{1}{N} \sum_{\mathbf{x}_{i} \in X} \mathbf{x}_{i}
$$

where $N$ is the total number of sample data, $\mathbf{x}_{i}$ is a feature vector to represent $i^{\text {th }}$ sample, and $X$ is the set of feature vectors for $N$ samples. Normalized correlation between features $i$ and $j$ is defined by

$$
\hat{C}(i, j)=\frac{C(i, j)}{\sqrt{C(i, i) \times C(j, j)}} .
$$

In the figure, both the $x$ - and $y$-axis depict the 144 features. The light-colored areas in the figure indicate high correlation between features, and the dark-colored areas indicate low correlation. If any feature correlates only with itself, only the diagonal elements will be light-colored. The off-diagonal light-colored areas in Figure 1(a) indicate that this image dataset exhibits not only a high correlation of features within the same media source, but also between certain features from different media sources (e.g., color and texture). Color and texture are traditionally treated as orthogonal modalities, but this example shows otherwise. These correlated and even noisy "raw" features may affect the learning algorithm by obscuring the distributions of truly relevant and representative features. (The weighted-sum fusion rule cannot deal with these interdependencies.)

Figure 1(b) presents the feature correlation matrix after we applied both PCA and ICA to the data. The process yields 58 "improved" components. Although the components exhibit better independence, interdependencies between components still exist. Our work in this paper first deals with grouping components like these 58 into a smaller number of independent modalities to avoid the dimensionality curse. We then explore non-linear combinations of the modalities to improve the effective multimodal fusion.

\subsection{Contribution Summary}

As the main contribution of this work, we propose a multimodalfusion framework for multimedia data analysis. Given a list of features extracted from multiple media-sources, we tackle two core issues:

- Formulating independent feature modalities (Section 3).

- Fusing multiple modalities optimally (Section 4).

We carefully analyze the tradeoffs between three design factors that affect fusion performance: modality independence, curse of dimensionality, and fusion-model complexity. Through analytical and empirical studies on an image dataset and TREC-Video 2003 benchmarks, we show that a careful balance of the three design factors consistently leads to superior performance for multimodal fusion.

\section{RELATED WORK}

We discuss related work in modality identification and modality fusion.

\subsection{Modality Identification}

Let $D$ denote the number of modalities. Given $d_{1} \cdots d_{m}$ features extracted from $m$ media sources, respectively, prior modality identification work can be divided into two representative categories.

1. $D=1$, or treating all features as one modality. This approach does not require the fusion step. Goh et al. [14] used the raw color and texture features to form a high-dimensional feature vector for each image. Recently, statistical methods such as PCA and ICA have been widely used in the Computer Vision, Machine Learning, Signal Processing communities to denoise data and to identify independent information sources (e.g., $[8,15,21,32,34])$. In the multimedia community, the work of $[16,18]$ observed that audio and visual data of a video stream exhibit some statistical regularity, and that regularity can be explored for joint processing. Smaragdis et al. [27] proposed to operate on a fused set of audio/visual features and to look for combined subspace components amenable to interpretation. Vinokourov et al. [33] found a common latent/semantic space from multilanguage documents using independent component analysis for cross-language document retrieval. The major shortcoming of these works is that the curse of dimensionality arises, causing ineffective feature-to-semantics mapping and inefficient indexing [25]. (Please refer to [7, 11, 12] for the discussion of dimensionality-curse and why dimension reduction can greatly enhance the effectiveness of statistical analysis and the efficiency of query processing.)

2. $D=m$, or treating each source as one modality. This approach treats the features as $m$ modalities, with $d_{i}$ features in the $i^{t h}$ modality $(i=1, \cdots, m)$. Most work in image and video retrieval analysis (e.g., [2, 13, 26, 28, 31]) employs this approach. For example, the QBIC system [13] supported image queries based on combining distances from the color and texture modalities. Velivelli et al. [31] separated video features into audio and visual modalities. IBM video analysis [2] also regarded each media track (visual, audio, textual, etc.) as one modality. For each modality, these works trained a separate classification model, and then used the weightedsum rule to fuse a class-prediction decision. This modalitydecomposition method can alleviate the curse of dimensionality. However, since media sources are treated separately, the interdependencies between sources are left unexplored.

Our method is to apply independent component analysis on the raw feature sets to identify $k$ "independent" components. There- 


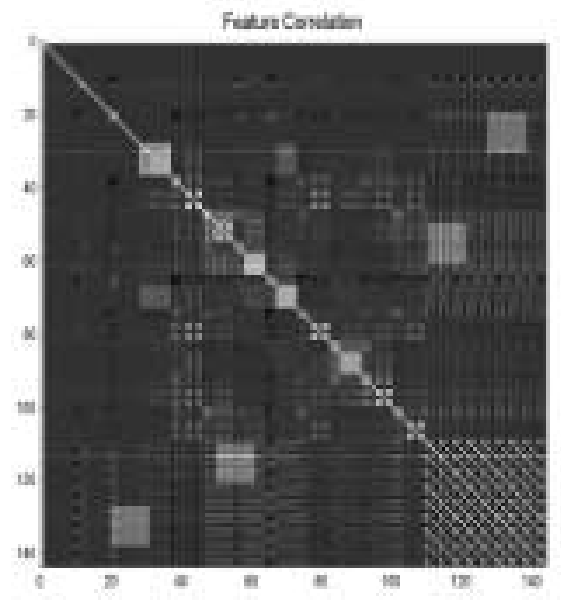

(a) Before PCA/ICA

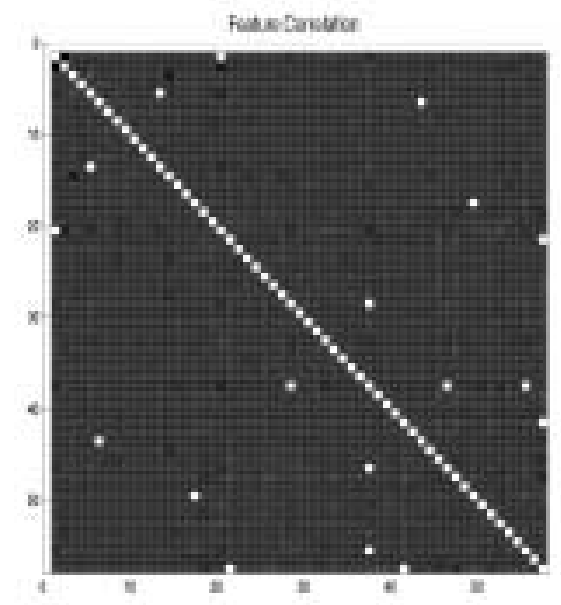

(b) After PCA/ICA

Figure 1: Feature Correlation Matrix.

after, we group these components into $D$ modalities to 1) minimize the dependencies between modalities, and 2) mitigate the dimensionality-curse problem.

\subsection{Modality Fusion}

Given that we have obtained $D$ modalities, we need to fuse $D$ classifiers, one for each modality, for interpreting data.

PCA and ICA cannot perfectly identify independent components for at least two reasons. First, like the way the $k$-means algorithm works, all well-known ICA algorithms (fixed-point algorithm [17], Infomax [1, 5], kernel canonical analysis [33], and kernel independent analysis [3]) need a good estimate of the number of independent components $k$ to find them effectively. Second, as we discussed in Section 1, ICA only performs the best attempt under some error-minimization criteria to find $k$ independent components. But the resulting components, as shown in Figure 1(b), may still exhibit interdependencies.

Now, given $D$ modalities, not entirely independent of each other, we need an effective fusion strategy. Various fusion strategies for multimodal information have been presented and were discussed in [20], including product combination, weighted-sum, voting, and min-max aggregation. Among them, product combination and weightedsum are by far the most popular fusion methods.

1. Product combination. Supposing that $D$ modalities are independent of each other, and we can estimate posterior probability for each modality accurately, the product-combination rule is the optimal fusion model from the Bayesian perspective. However, in addition to the fact that we will not have $D$ truly independent modalities, we generally cannot estimate posterior probability with high accuracy. The work of [29] concluded that the product-combination rule works well only when the posterior probability of individual classifiers can be accurately estimated. In a multimedia data-understanding task, we often assert similarity between data based on our beliefs. (E.g., one can "believe" two videos to be $87 \%$ similar or $90 \%$ similar. This estimate does not come from classical probability experiments, so the sum of beliefs may not be equal to one.) Because of this subjective process, and because the product-combination rule is highly sensitive to noise, this strategy is not appropriate.
2. Weighted-sum. The weighted-sum strategy is more tolerant to noise because sum does not magnify noise as severely as product. Weighted-sum (e.g., [30]) is a linear model, not equipped to explore the interdependencies between modalities. Recently, Yan and Hauptmann [35] presented a theoretical framework for bounding the average precision of a linear combination function in video retrieval. Concluding that the linear combination functions have limitations, they suggested that non-linearity and cross-media relationships should be introduced to achieve better performance.

In this work, we propose a super-kernel scheme, which can fuse multimodal information non-linearly to explore the cross-modality relationship.

\section{INDEPENDENT MODALITY ANALYSIS}

In this section, we present our approach to transform $m$ raw features to $D$ modalities. Given input in the form of an $m \times n$ matrix $X$ ( $n$ denotes the number of training instances), our independent modality analysis procedure produces $M_{1} \cdots M_{D}$ modalities. The procedure consists of the following three steps:

1. Run principal component analysis (PCA) on $X$ to remove noise and reduce the feature dimensionality. Let $U$ denote the matrix containing the first $k$ eigenvectors. The PCA representation of zero-mean feature vectors $X$ is defined as $U^{T} X$

2. Run independent component analysis (ICA) on the PCA output $U^{T} X$ to obtain estimates of independent feature components $S$ and an estimate of a mixing matrix $W$. We can recover the independent components by computing $S=W U^{T} X$.

3. Run independent modality grouping (IMG) on $S$ to form independent modalities $M_{1}, \cdots, M_{D}$.

\subsection{PCA}

PCA has been frequently used as a technique for removing noises and redundancies between feature dimensions [19]. PCA projects the original data to a lower dimensionality space such that the variance of the data is best maintained. Let's assume that we have $n$ samples, $\left\{\mathbf{x}_{1}, \cdots, \mathbf{x}_{n}\right\}$, and each $\mathbf{x}_{i}$ is an $m$-dimensional vector. 


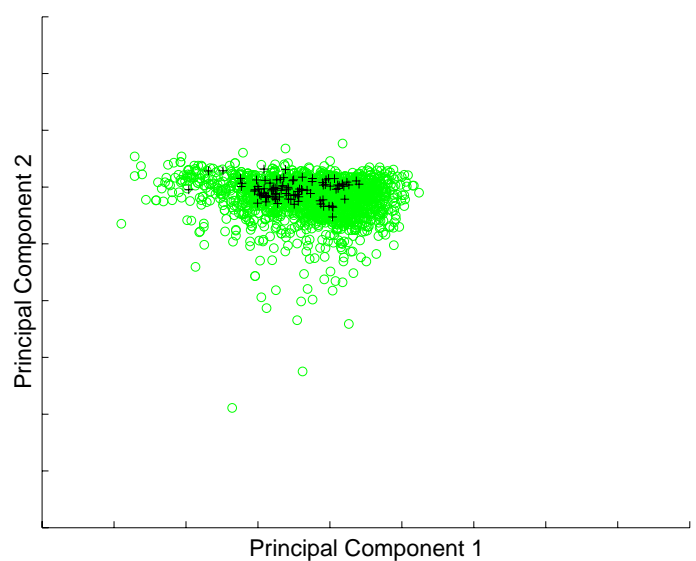

(a) PCA

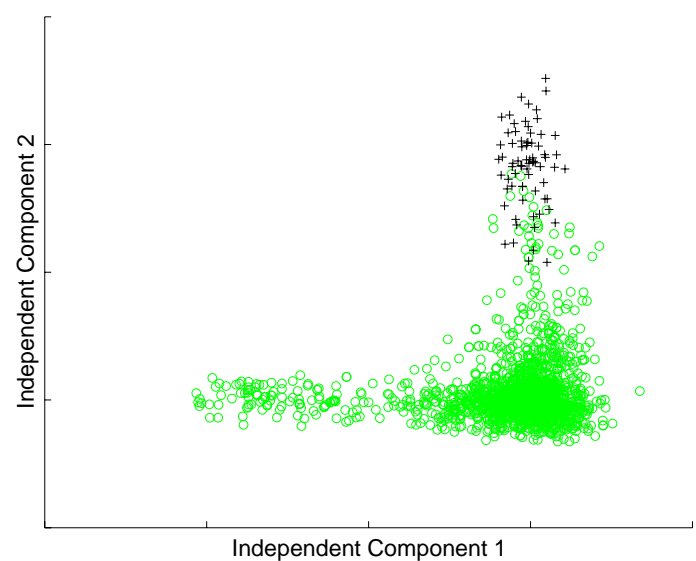

(b) ICA

Figure 2: Scatter Plots of the $2 k$ Image Dataset.

We can represent the $n$ samples as a matrix $X_{m \times n}$. It is known in linear algebra that any such matrix can be decomposed in the following form (known as singular value decomposition or SVD):

$$
X=U D V^{T}
$$

where matrices $U_{m \times p}$ and $V_{n \times p}$ represent orthonormal basis vectors matrices (eigenvectors of the symmetric matrix $X X^{T}$ and $X^{T} X$ ), with $p$ as the number of largest principal components. The $D_{p \times p}$ matrix is a diagonal matrix, and the diagonal elements of $D$ are the eigenvalues of $X X^{T}$ and $X^{T} X$. Consider the projection onto the subspace spanned by the $p$ largest principal components (PC's), i.e., $U^{T} X$.

\subsection{ICA}

Compared to PCA, the spirit of ICA is to find statistically independent hidden sources from a given set of mixture signals. Both ICA and PCA project data matrices into components in different spaces. However, the goals of the two methods are different. PCA finds the uncorrelated components of maximum variance. It is ideal for compressing data into a lower-dimensional space by removing the least significant components. ICA finds the statistically independent components. ICA is the ideal choice for separating mixed signals and finding the most representative components.

To formalize an ICA problem, we assume that there are $k$ unknown independent components $S=\left\{s_{1}, \cdots, s_{k}\right\}$. What we observe is a set of $m$-dimensional samples $\left\{\mathbf{x}_{1}, \cdots, \mathbf{x}_{n}\right\}$, which are mixture signals coming from $k$ independent components, $k \leq m$. We can represent all the observation data as a matrix $X_{m \times n}$. A linear mixture model can be formulated as:

$$
X=A S
$$

where $A_{m \times k}$ is a mixing matrix. Our goal is to find $W=A^{-1}$; therefore given training set $X$, we can recover the independent components (IC's) through the transformation of $S=W X$.

ICA establishes a common latent space for the media, which can be viewed as a method for learning the inter-relations between the involved media [23, 27]. For multimedia data, observation data $\mathbf{x}_{i}$ usually contains features coming from more than one medium. The different independent components $\left\{s_{1}, \cdots, s_{k}\right\}$ provide a meaningful segmentation of the feature space. The $k^{t h}$ column of $W^{-1}$ constitutes the original multiple features associated with the $k^{t h}$ independent component. These independent components can provide a better interpretation for multimedia data. Figures 2(a) and (b) show the scatter plots of the $2 k$ image dataset, projected to a two-dimensional subspace identified by the first two principal components and the first two independent components. Dark points correspond to the class of tools (one of the 14 classes), and green (light) points correspond to the other 13 classes. Compared with PC's in Figure 2(a), IC's found from ICA in Figure 2(b) can better separate data from different semantic classes. Figure 2(b) strongly suggests an ICA interpretation to differentiate semantics. The main attraction of ICA is that it provides unsupervised groupings of data that have been shown to be well aligned with manual grouping in different media [15]. The representative and non-redundant feature representations form a solid base for later processing.

Lacking any prior information about the number of independent components, ICA algorithms usually assume that the number of independent components is the same as the dimension of observed mixtures, that is, $k=m$. PCA technique can be used as preprocessing to ICA to reduce noise in the data and control the number of independent components [4]. Then ICA is performed on the main eigenvectors of PCA representations ( $k=p$, where $p$ is the number of PC's) to determine which PC's actually are independent and which should be grouped together as parts of a multidimensional component. Finally, the independent components are recovered by computing $S=W U^{T} X$.

\subsection{IMG}

As discussed in Sections 1 and 2, though ICA makes a best attempt to find independent components, the resulting $k$ components might not be independent, and the number of components can be too large to face the challenge of "dimensionality curse" during the statistical-analysis and query-processing phrases. IMG aims to remedy these two problems by grouping $k$ components into $D$ modalities.

We divide $k$ components into $D$ groups to satisfy two requirements: 1) the correlation between modalities is minimized, and 2) the number of features in each modality is not too large. The first requirement maximizes modality independence. The second requirement avoids the problem of curse-of-dimensionality. To decide on $D$, we place a soft constraint on the number of components that a modality can have. We set the soft constraint as 30 because several prior works $[7,11,12]$ indicate that when the number of dimensions exceeds 20 to 30 , the curse starts to kick in. Since only the data can tell us exactly at what dimension the curse starts to take effect, the selection of $D$ must go through a cross-validation 


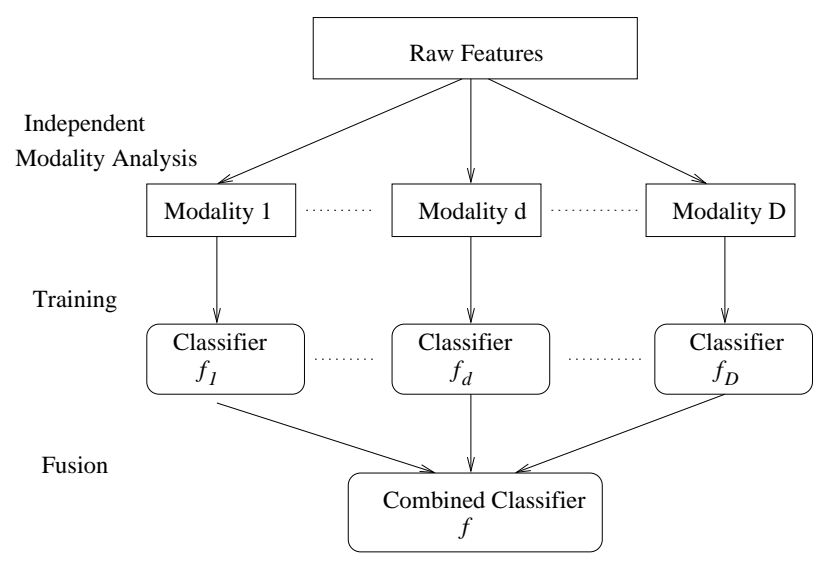

Figure 3: Fusion Architecture.

process: we pick a small number of candidate $D$ values and rely on experiments to select the best $D$.

For a given $D$, we employ a clustering approach to divide $k$ into $D$ groups. Ding et al. [10] provided theoretical analysis to show that minimizing inter-subgraph similarities and maximizing intra-subgraph similarities always lead to more balanced graph partitions. Thus, we apply minimizing inter-group feature correlation and maximizing intra-group feature correlation as our featuregrouping criteria to determine independent modalities. Suppose we have $D$ modalities $M_{1}, \cdots, M_{D}$, each containing a number of feature components. The inter-group feature correlation between two modalities $M_{i}$ and $M_{j}$ is defined as

$$
C\left(M_{i}, M_{j}\right)=\sum_{\forall S_{i} \in M_{i}, \forall S_{j} \in M_{j}} C\left(S_{i}, S_{j}\right),
$$

where $S_{i}$ and $S_{j}$ are features belonging to modalities $M_{i}$ and $M_{j}$ respectively, and $C\left(S_{i}, S_{j}\right)$ is the normalized feature correlation between $S_{i}$ and $S_{j} . C\left(S_{i}, S_{j}\right)$ can be calculated using Equation 1 and Equation 2. The intra-group feature correlation within modality $M_{i}$ is defined as

$$
C\left(M_{i}\right)=C\left(M_{i}, M_{i}\right) .
$$

To minimize inter-group feature correlation while maximizing intragroup feature correlation at the same time, we can formulate the following objective function for grouping all the features into $D$ modalities,

$$
\min \sum_{\substack{i=1 \\ j>i}}^{D}\left[\frac{C\left(M_{i}, M_{j}\right)}{C\left(M_{i}\right)}+\frac{C\left(M_{i}, M_{j}\right)}{C\left(M_{j}\right)}\right] .
$$

Solving this objective function yields $D$ modalities, with minimal inter-modality correlation and balanced features in each modality. The computational complexity is $O\left(k^{2}\right)$.

\section{SUPER-KERNEL FUSION}

Once $D$ modalities have been identified by our independent modality analysis, we need to fuse multimodal information optimally. Suppose we train for the $d^{\text {th }}$ modality classifier $f_{d}$. We need to combine these $D$ classifiers to perform class prediction for query instance $\mathbf{x}_{q}$. The fusion architecture is depicted in Figure 3 .

After $f_{d}, d=1 \cdots D$ have been trained, the information can be fused in several ways. Let $f$ denote the fused classification func- tion. The product-combination rule can be formulated as

$$
f=\prod_{d=1}^{D} f_{d}
$$

And the most widely used weighted-sum rule can be depicted as

$$
f=\sum_{d=1}^{D} \mu_{d} f_{d}
$$

where $\mu_{d}$ is the weight for individual classifier $f_{d}$. As we have discussed in Section 2, both these popular models suffer from several shortcomings, including being sensitive to prediction error and being limited by the linear-model complexity. (Please consult Section 2 for detailed discussion.) To overcome these shortcomings, we propose using super-kernel fusion to aggregate $f_{d}$ 's.

The algorithm of super-kernel fusion is summarized in Figure 4, which consists of the following three steps:

1. Train individual classifiers $\left\{f_{d}\right\}$. The inputs to the algorithm are the $n$ training instances $\left\{\mathbf{x}_{1}, \cdots, \mathbf{x}_{n}\right\}$ and their corresponding labels $\left\{y_{1}, \cdots, y_{n}\right\}$. After the independent modality analysis (IMA), the $m$-dimensional features are divided into $D$ modalities. Each training instance $\mathbf{x}_{i}$ is represented by $\left\{\mathbf{x}_{i}^{1}, \cdots, \mathbf{x}_{i}^{D}\right\}$, where $\mathbf{x}_{i}^{d}$ is the feature representation for $x_{i}$ in $d^{\text {th }}$ modality. All the training instances are divided into $D$ matrices $\left\{M_{1}, \cdots, M_{D}\right\}$, where each $M_{d}$ is an $n \times\left|M_{d}\right|$ matrix, and $\left|M_{d}\right|$ is the number of features in $d^{t h}$ modality $(d=1 \cdots D)$. To train classifier $f_{d}$, we use $M_{d}$ and the label information. Though many learning algorithms can be employed to train $f_{d}$, we employ an SVM as our base-classifier because of its effectiveness. For training each $f_{d}$, the kernel function and kernel parameters are carefully chosen via cross validation (steps $1-3$ in Figure 4).

2. Estimate posterior probability. Once we have trained $D$ classifiers for the $D$ modalities, we create a super-kernel matrix $K$ for modality fusion. This matrix is created by passing each training instance to each of the $D$ classifiers to estimate its posterior probability. We use Platt's formula [24] to convert an SVM score to probability. As a result of this step, we obtain an $n \times D$ matrix consisting of $n$ entries of $D$ classprediction probability (steps $4-6$ in Figure 4 ).

3. Fuse the classifiers. The super-kernel algorithm treats $K$ a matrix of $n$ training instances, each with a vector of $D$ elements. Next, we again employ SVMs to train the superclassifier. The inputs to SVMs include $K$, training labels, a selected kernel function, and kernel parameters. At the end of the training process, we yield function $f$ to perform class prediction. The complexity of the fusion model depends on the kernel chosen. For instance, we can select a polynomial, $\mathrm{RBF}$ or Laplacian function (steps $7-8$ in Figure 4).

Finally, once the class-prediction function $f$ has been trained, we can use the function to predict the class membership of a query point $\mathbf{x}_{q}$. Assume $\mathbf{x}_{q}$ is an $m$-dimensional feature vector in original feature space, we can convert it to an ICA feature representation $W U^{T} \mathbf{x}_{q}$, where $W$ and $U$ are transformation matrices obtained from PCA and ICA process, respectively (Section 3). Then, $W U^{T} \mathbf{x}_{q}$ is further divided into $D$ modalities (information obtained from the IMG process), named as $\left\{\mathbf{x}_{q}^{1}, \cdots, \mathbf{x}_{q}^{D}\right\}$. The class-prediction function for query point $\mathbf{x}_{q}$ can be written as

$$
\hat{y}_{q}=f\left(f_{1}\left(\mathbf{x}_{q}^{1}\right), \cdots, f_{d}\left(\mathbf{x}_{q}^{D}\right)\right) .
$$




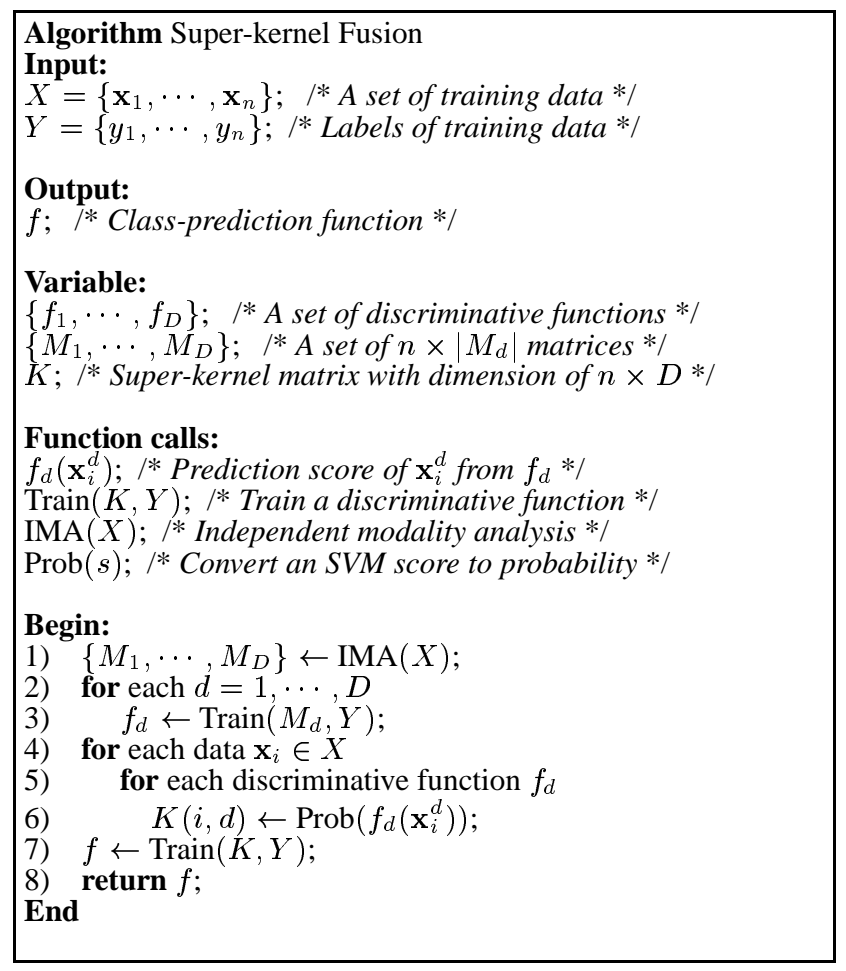

Figure 4: Super-kernel Fusion Algorithm.

\section{EXPERIMENTS}

Our experiments were designed to evaluate the effectiveness of using independent modality analysis and multimodal kernel fusion to determine the optimal multimodal information fusion for multimedia data retrieval. Specifically, we wanted to answer the following questions:

1. Can independent modality analysis improve the effectiveness of multimedia data analysis?

2. Can super-kernel fusion improve fusion performance?

We conducted our experiments on two real-world datasets: one is a $2 k$ image dataset, and the other is TREC-2003 video track benchmark. We randomly selected a percentage of data from the dataset to be used as training examples. The remaining data were used for testing. For each dataset, the training/testing ratio was empirically chosen via cross-validation so that the sampling ratio worked best in our experiments. To perform independent modality analysis, we applied traditional PCA and ICA algorithms ${ }^{2}$ onto the given features (including all the training and testing data) to get the independent components following the steps described in Section 3. To perform class prediction, we employed the one-per-class (OPC) ensemble [9], which trains all the classifiers, each of which predicts the class membership for one class. The class prediction on a testing instance is decided by voting among all the classifiers. The results presented here were the average of 10 runs.

\section{Dataset \#1: $2 k$ image dataset.}

The image dataset was collected from the Corel Image CDs. Corel images have been widely used by the computer vision, image processing, and multimedia research communities for conducting var-

\footnotetext{
${ }^{2}$ InfoMax was chosen as our ICA algorithm because of its robustness, though other ICA algorithms could also be applied.
}

ious experiments. This set contains $2 k$ representative images from fourteen categories: architecture, bears, clouds, elephants, fabrics, fireworks, flowers, food, landscape, people, textures, tigers, tools, and waves. We tried different kernel functions, kernel parameters and training/testing ratios. Laplacian kernel with $\gamma=0.001$ and $80 \%$ of the dataset as training data gave us the best results on the experiments of using raw features. We used the Laplacian kernel with $\gamma=0.001$ for all subsequent experiments on this $2 k$ image dataset. We randomly picked $80 \%$ of images for training and the remaining $20 \%$ were used for testing data. For each image, we extracted 144 features (documented in [22]) including color and texture features. This small dataset is used to provide insights into understanding the effectiveness of our methods, and the tradeoffs between design factors.

Dataset \#2: TREC-2003 Video Track.

TREC-2003 video track used 133 hours digital video (MPEG-1) from $\mathrm{ABC}$ and $\mathrm{CNN}$ news. The task is to detect the presence of the specified concept in video shots. The ground-truth of the presence of each concept was assumed to be binary (either present or absent in the data). Sixteen concepts are defined in the benchmark, including airplane, animal, building, female speech, madeleine albright, nature vegetation, news subject face, news subject monologue, NIST non-studio setting, outdoors, people, physical violence, road, sport event, vehicle, and weather news. The video concept detection benchmark is summarized as follows: $60 \%$ of the video shots were randomly chosen from the corpus to be used solely for the development of classifiers. The remaining $40 \%$ were used for concept validation ${ }^{3}$. RBF kernels with $\gamma=0.0001$ gave us the best results on the experiments, so we used the same parameter settings in all subsequent experiments on this video dataset. For each video shot, we extracted a number of features [2]: Color histogram, Edge orientation histogram, Color correlogram, Co-occurrence texture, Motion vector histogram, Visual perception texture, and Speech.

\subsection{Evaluation of Modality Analysis}

The first set of experiments examined the effectiveness of independent modality analysis on the $2 k$ image dataset. Table 1 compares five methods based on the classification accuracy results of 14 concepts: original 144 dimensional features before any analysis (RAW), super-kernel fusion using 108 dimensional color features and 36 dimensional texture features as 2 modalities (SKF), 58 dimensional features after PCA (PCA), 58 dimensional features after ICA (ICA) and super-kernel fusion after IMG (IMG+SKF). As shown in the table, treating color and texture as two modalities improved the accuracy by around $1.0 \%$ compared to using raw feature representation. However, the accuracy was $4.0 \%$ lower than superkernel fusion after IMG. This observation indicates that improvement can be made by using super-kernel fusion to cover the interdependency relationship between features. Moreover, after analyzing the statistical relationships between feature dimensions and getting rid of noise, super-kernel fusion can improve the performance much more. PCA improved accuracy by around $1.0 \%$ compared to the original feature format by reducing noise from features. ICA worked better than PCA, improving accuracy by $2.5 \%$ compared to the original feature format. However, the improvement is not significant, compared to the performance of super-kernel fusion after IMG. Independent modality analysis plus super-kernel fusion improved classification accuracy around $5.0 \%$ compared to the original feature representation. The result shows that the feature sets

\footnotetext{
${ }^{3}$ IBM research center won most of the best concept models in the final TREC-2003 video concept competition. For the purpose of comparison, we employed the same training and testing data used by IBM.
} 


\begin{tabular}{l|ccccc}
\hline CATEGORY & RAW & SKF & PCA & ICA & IMG+SKF \\
\hline \hline ARCHITECTURE & 88.00 & 89.95 & 90.77 & 95.38 & $\mathbf{9 6 . 9 2}$ \\
BEARS & 74.70 & 76.72 & 75.00 & 75.00 & $\mathbf{8 1 . 5 6}$ \\
CLOUDS & 84.60 & 87.61 & 87.27 & 90.91 & $\mathbf{9 2 . 3 2}$ \\
ELEPHANTS & 83.90 & 84.67 & 84.83 & 87.21 & $\mathbf{8 9 . 9 1}$ \\
FABRICS & 85.10 & 85.90 & 87.22 & 87.82 & $\mathbf{8 7 . 9 3}$ \\
FIREWORKS & 93.50 & 95.69 & 94.91 & 96.46 & $\mathbf{9 9 . 5 0}$ \\
FLOWERS & 91.30 & $\mathbf{9 5 . 5 3}$ & 92.21 & 93.49 & 95.23 \\
FOOD & 92.20 & 95.58 & 93.36 & 95.76 & $\mathbf{9 7 . 4 8}$ \\
LANDSCAPE & 78.80 & 72.79 & 79.48 & 79.63 & $\mathbf{8 1 . 8 2}$ \\
PEOPLE & 82.30 & 85.50 & 87.45 & 86.27 & $\mathbf{8 9 . 3 6}$ \\
TEXTURES & $\mathbf{9 6 . 5 0}$ & 91.62 & 91.22 & 95.00 & 96.30 \\
TIGERS & 91.50 & 92.34 & 91.13 & 92.64 & $\mathbf{9 4 . 8 0}$ \\
TOOLS & 99.50 & 98.15 & 96.74 & $\mathbf{1 0 0 . 0 0}$ & 99.20 \\
WAVES & 86.10 & 89.49 & 84.71 & 87.27 & $\mathbf{9 1 . 4 2}$ \\
\hline \hline Average & 87.71 & 88.82 & 88.66 & 90.20 & $\mathbf{9 2 . 7 0}$ \\
\hline
\end{tabular}

Table 1: Classification Accuracy (\%) of Image Dataset.

from independent modality analysis can better interpret the concepts, and super-kernel fusion can further incorporate information from multiple modalities. Next, we evaluated how to select optimal $D$ and compared super-kernel fusion with other fusion methods.

\subsection{Evaluation of Multimodal Kernel Fusion}

The second set of experiments evaluated kernel fusion methods of combining multiple modalities. We grouped the "independent" components after PCA/ICA into independent modalities and trained individual classifiers for each modality. We evaluated the effectiveness of multimodal kernel fusion on the $2 k$-image dataset and TREC-2003 video benchmark.

The optimal number of independent modalities $D$ was decided by considering the tradeoff between dimensionality-curse and feature interdependency. Once $D$ had been determined, feature components were grouped using the IMG algorithm in Section 3.3. When $D=1$, all the feature components were treated as one vector representation, suffering from the curse of dimensionality. When $D$ became larger, the curse of dimensionality was alleviated, but intermodality correlation increased ${ }^{4}$. From our 58 -dimensional feature data, the optimal modality $D$ is 2 or 3 , which enjoys the highest class-prediction accuracy. Table 2 shows the optimal $D$ for different concepts (the second column).

Next, we compared different fusion models. Table 2 compares the class-prediction accuracy of product combination (PC), linear combination (LC), and super-kernel fusion (SKF). $D$ indicates the number of independent modalities that the 58 independent components have been divided into. We found that super-kernel fusion performed on average $6.5 \%$ better than product-combination models and $4.5 \%$ better than linear-combination models. Note that the worst results were achieved when using the product rule, $2.0 \%$ worse than linear-combination models and $6.5 \%$ worse than those of super-kernel fusion. The reason is that if any of the classifiers reports the correct class's posterior probability as zero, the output will be zero, and the correct class cannot be identified. Therefore, the final result reported by the combiner in such cases is either a wrong class (worst case) or a reject (when all of the classes are assigned a zero posterior probability).

Finally, we conducted fusion experiments on the video dataset. For this TREC video dataset, we got only probability outputs from single-modality classifiers through IBM. Therefore, we evaluated only fusion schemas on this video dataset. Table 3 compares the

\footnotetext{
${ }^{4}$ The inter-modality correlation for all the $D$ modalities is the summation of inter-modality correlations between every pair of modalities, which is $\sum_{i=1 j>i}^{D} C\left(M_{i}, M_{j}\right)$.
}

\begin{tabular}{l|c|ccc}
\hline CATEGORY & D & PC & LC & SKF \\
\hline \hline ARCHITECTURE & 2 & 96.40 & 96.53 & $\mathbf{9 6 . 9 2}$ \\
BEARS & 2 & 76.10 & 75.35 & $\mathbf{8 1 . 5 6}$ \\
CLOUDS & 3 & 82.71 & 89.77 & $\mathbf{9 2 . 3 2}$ \\
ELEPHANTS & 2 & 86.11 & 80.91 & $\mathbf{8 9 . 9 1}$ \\
FABRICS & 2 & 85.11 & 87.46 & $\mathbf{8 7 . 9 3}$ \\
FIREWORKS & 2 & 97.63 & 99.13 & $\mathbf{9 9 . 5 0}$ \\
FLOWERS & 3 & 82.29 & 86.14 & $\mathbf{9 5 . 2 3}$ \\
FOOD & 2 & 93.45 & 89.53 & $\mathbf{9 7 . 4 8}$ \\
LANDSCAPE & 2 & 77.55 & 74.24 & $\mathbf{8 1 . 8 2}$ \\
PEOPLE & 2 & $\mathbf{9 0 . 7 1}$ & 89.57 & 89.36 \\
TEXTURES & 2 & 74.51 & 94.27 & $\mathbf{9 6 . 3 0}$ \\
TIGERS & 3 & 87.31 & $\mathbf{9 5 . 0 0}$ & 94.80 \\
TOOLS & 2 & 91.48 & 94.20 & $\mathbf{9 9 . 2 0}$ \\
WAVES & 2 & 86.92 & 82.13 & $\mathbf{9 1 . 4 2}$ \\
\hline \hline Average & 2.3 & 86.31 & 88.16 & $\mathbf{9 2 . 7 0}$ \\
\hline
\end{tabular}

Table 2: Classification Accuracy (\%) of Image Dataset.

best results from IBM (IBM), product combination (PC), linear combination (LC), and super-kernel fusion (SKF) based on Average Precision of video concept detection. The numbers of modalities $D$ for sixteen concepts ranged from 3 to 6 (compared to the $2 k$ image dataset, this video dataset extracted features with much higher dimension. To avoid the dimensionality curse, we need larger $D$ ). Here we chose the NIST Average Precision (the sum of the precision at each relevant hit in the hitlist divided by the total number of relevant documents in the collection) as the evaluation criteria. Average Precision (AP) was used by NIST to evaluate retrieval systems in TREC-2003 video track competition. For TREC2003 video track, a maximum of 1,000 entries ${ }^{5}$ were returned and ranked according to the highest probability of detecting the presence of the concept. The ground-truth of the presence of each concept was assumed to be binary (either present or absent in the data). For the 16 concepts in TREC-2003 video benchmark, super-kernel fusion performed around $5.2 \%$ better than the linear-combination models on average, $11.3 \%$ better than product-combination models. IBM results were obtained from fusion across modalities and semantics [2]. Super-kernel fusion, which is fusion across modalities only, performed around $2.0 \%$ better than the best results provided by IBM.

\subsection{Observations}

After our extensive empirical studies on the two datasets, we can answer the questions proposed at the beginning of this section. 1. To deal with high-dimensional features from multiple media

\footnotetext{
${ }^{5}$ This number was chosen in the IBM's work [2] for evaluation.
} 


\begin{tabular}{l|cccc}
\hline CONCEPT & IBM & PC & LC & SKF \\
\hline \hline AIRPLANE & $\mathbf{2 4 . 9 3}$ & 10.60 & 23.52 & 24.31 \\
ANIMAL & 6.09 & 6.75 & $\mathbf{8 . 5 9}$ & 8.2 \\
BUILDING & 8.02 & 7.92 & 4.68 & $\mathbf{8 . 4 2}$ \\
FEMALE SPEECH & 67.23 & 49.10 & 67.23 & $\mathbf{6 7 . 3 3}$ \\
MADELEINE ALBRIGHT & $\mathbf{4 7 . 4 1}$ & 16.54 & 33.93 & 43.27 \\
NATURE VEGETATION & 37.84 & 31.02 & 33.65 & $\mathbf{3 9 . 3 9}$ \\
NEWS SUBJECT FACE & $\mathbf{8 . 1 2}$ & 1.37 & 7.89 & 7.05 \\
NEWS SUBJECT MONO. & $\mathbf{2 0 . 4 1}$ & 3.1 & 8.87 & 13.48 \\
NIST NON-STUDIO & 69.1 & 69.65 & 66.38 & $\mathbf{6 9 . 8 8}$ \\
OUTDOORS & 65.16 & $\mathbf{6 9 . 8 1}$ & 53.87 & 66.16 \\
PEOPLE & 11.82 & 12.95 & 16.41 & $\mathbf{1 8 . 9 1}$ \\
PHYSICAL VIOLENCE & $\mathbf{3 . 0 4}$ & 1.06 & 1.42 & 1.8 \\
ROAD & 10 & 7.72 & $\mathbf{1 2 . 4 2}$ & 8.38 \\
SPORT EVENT & 48.45 & 24.20 & 40.49 & $\mathbf{5 2 . 8}$ \\
VEHICLE & $\mathbf{2 0 . 8 1}$ & 14.05 & 15.63 & 16.54 \\
WEATHER NEWS & 53.64 & 29.73 & 53.64 & $\mathbf{8 6 . 7}$ \\
\hline \hline Average & 31.38 & 22.28 & 28.04 & $\mathbf{3 3 . 2 9}$ \\
\hline
\end{tabular}

Table 3: AP (\%) of Video Concept Detection.

sources, it is necessary to do statistical analysis to reduce noise and find the most representative feature-components. Independent modality analysis can improve the effectiveness of multimedia data analysis by achieving a tradeoff between dimensionality curse and modality independency.

2. Super-kernel fusion is superior in its performance because its high model complexity can explore interdependencies between modalities.

\section{CONCLUSION}

In this paper, we have proposed a framework of optimal multimodal information fusion for multimedia data analysis. First, we constructed statistically independent modalities from the given feature set from multiple media sources. Next, we proposed superkernel fusion to learn the optimal combination of multimodal information. We carefully analyzed the tradeoffs between three design factors that affect fusion performance: modality independence, curse of dimensionality, and fusion-model complexity. Our extensive empirical studies show that our methods achieved markedly improved performance on a $2 k$ image dataset and TREC-Video 2003 benchmarks.

From the experimental results, we observe that different concepts may be best depicted by different combinations of modalities. We will extend this work to investigate concept-dependent multimodal fusion schemes.

\section{Acknowledgement}

The first two authors are supported by NSF grants Career IIS-0133802 and ITR IIS-0133802.

\section{REFERENCES}

[1] S. Amari, A. Cichocki, and H. H. Yang. A new learning algorithm for blind signal separation. Advances in Neural Information Processing Systems, 8:757-763, 1996.

[2] A. Amir, H. W, G. Iyengar, C.-Y.Lin, M. Naphade, A. Natsev, C. Neti, H. J Nock, J. R. Smith, B. L. Tseng, Y. Wu, and D. Zhang. IBM research TRECVID-2003 system. NIST Text Retrieval Conf. (TREC), 2003.

[3] F. R. Bach and M. I. Jordan. Kernel independent component analysis. Machine Learning Research, 3:1-48, 2002.

[4] M. S. Bartlett, H. M. Lades, and T. J. Sejnowski. Independent component representation for face recognition. SPIE Conf. on Human Vision and Electronic Imaging III, 3299:528-539, 1998.

[5] A. J. Bell and T. J. Sejnowski. An information-maximization approach to blind separation and blind deconvolution. Neural Computation, 7(6):1129-1159, 1995.

[6] R. Bellman. Adaptive control processes. Princeton, 1961.
[7] K. Beyer, J. Goldstein, R. Ramakrishnan, and U. Shaft. When is "nearest neighbor" meaningful? International Conference on Database Theory, pages 217-235, 1999.

[8] M. L. Cascia, S. Sethi, and S. Sclaroff. Combining textual and visual cues for content-based image retrieval on the world wide web. IEEE Workshop on Content-based Access of Image and Video Libaries, pages 24-28, 1998.

[9] T. Dietterich and G. Bakiri. Solving multiclass learning problems via error-correcting output codes. Artifical Intelligence Research, 2:263-286, 1995.

[10] C. H. Q. Ding, X. He, H. Zha, M. Gu, and H. D. Simon. A min-max cut algorithm for graph partitioning and data clustering. IEEE International Conference on Data Mining, pages 107-114, 2001.

[11] D. L. Donoho. High-dimensional data analysis: The curses and blessings of dimensionality. American Math. Society Lecture-Match Challenges of the 21st Century, 2000.

[12] R. Fagin, A. Lotem, and M. .Naor. Optimal aggregation algorithms for middleware. ACM Symposium on Principles of Database Systems, 2001.

[13] M. Flickner, H. Sawhney, W. Niblack, J. Ashley, Q. Huang, B. Dom, M. Gorkani, J. Hafner, D. Lee, D. Petkovic, D. Steele, and P. Yanker. Query by image and video content: the QBIC system. Intelligent multimedia information retrieval, pages 7-22, 1997.

[14] K. Goh, E. Chang, and K.-T. Cheng. SVM binary classifier ensembles for multi-class image classification. ACM International Conference on Information and Knowledgement Management (CIKM), pages 395-402, 2001.

[15] L. Hansen, J. Larsen, and T. Kolenda. On independent component analysis for multimedia signals. Multimedia Image and VideoProcessing, CRC Press, 2000.

[16] J. Hershey and J. Movellan. Using audio-visual synchrony to locate sounds. Advances in Neural Information Processing Systems 12. MIT Press, Cambridge MA, 2001.

[17] A. Hyvarinen and E. Oja. A fast fixed-point algorithm for independent component analysis. Neural Computation archive, 9(7):1483-1492, 1997.

[18] J. F. III, T. Darrell, W. Freeman, and P. Viola. Learning joint statistical models for audio-visual fusion and segregation. Advances in Neural Information Processing Systems 13. MIT Press, Cambridge MA, 2000.

[19] I. Joliffe. Principal component analysis. Springer-Verlag, New York, 1986

[20] J. Kittler, M. Hatef, and R. P. W. Duin. Combining classifiers. Intl. Pattern Recognition, pages 897-901, 1996.

[21] T. Kolenda, L. K. Hansen, J. Larsen, and O. Winther. Independent component analysis for understanding multimedia content. IEEE Workshop on Neural Networks for Signal Processing, pages 757-766, 2002.

[22] B. Li and E. Chang. Discovery of a perceptual distance function for measuring image similarity. ACM Multimedia Journal Special Issue on Content-Based Image Retrieval, 8(6):512-522, 2003.

[23] A. S. Lukic, M. N. Wernick, L. K. Hansen, and S. C. Strother. An ICA algorithm for analyzing multiple data sets. IEEE Int. Conf. on Image Processing, pages 821-824, 2002.

[24] J. Platt. Probabilistic outputs for support vector machines and comparison to regularized likelihood methods. Advances in Large Margin Classifiers, MIT Press, pages 61-74, 2000.

[25] Y. Rui, T. S. Huang, and S. F. Chang. Image retrieval: Past, present, and future International Symposium on Multimedia Information Processing, 1997.

[26] Y. Rui, T. S. Huang, and S. Mehrotra. Content-based image retrieval with relevance feedback in mars. IEEE International Conference on Image, 1997.

[27] P. Smaragdis and M. Casey. Audio/visual independent components. International Symposium on Independent Component Analysis and Blind Source Separation, pages 709-714, 2003.

[28] J. R. Smith and S. F. Chang. Automatic image retrieval using color and texture. IEEE Trans Pattern Anal Mach Intell, 1996.

[29] D. M. J. Tax, M. V. Breukelen, R. P. W. Duin, and J. Kittler. Combing multiple classifiers by averaging or by multiplying. Pattern Recognition, 33:1475-1485, 2000.

[30] K. M. Ting and I. H. Witten. Issues in styacked generalization. Artificial Intelligence Research, 10:271-289, 1999.

[31] A. Velivelli, C. W. Ngo, and T. S. Huang. Detection of documentarty scene changes by audio-visual fusion. International conference on Image and video retrieval, pages 227-237, 2003.

[32] A. Vinokourov, D. R. Hardoon, and J. Shawe-Taylor. Learning the semantics of multimedia content with application to web image retrieval and classification. Fourth International Symposium on Independent Component Analysis and Blind Source Separation, 2003.

[33] A. Vinokourov, J. Shawe-Taylor, and N. Cristianini. Inferring a semantic representation of text via cross-language correlation analysis. Advances of Neural Information Processing, 2002.

[34] T. Westerveld. Image retrieval: Content versus context. Content-Based Multimedia Information Access, RIAO, 2000.

[35] R. Yan and A. G. Hauptmann. The combination limit in multimedia retrieval. ACM Multimedia, 2003. 